\title{
DESPISTAJE DE LA RETINOPATÍA DEL PREMATURO. NUESTRA EXPERIENCIA SOBRE LOS LÍMITES DE PESO AL NACER, EDAD GESTACIONAL Y OTROS FACTORES DE RIESGO
}

\author{
SCREENING FOR RETINOPATHY OF PREMATURITY. OUR \\ EXPERIENCE ABOUT LIMITS OF BIRTH WEIGHT, POST- \\ CONCEPTIONAL AGE AND OTHERS RISK FACTORS
}

\author{
RODRÍGUEZ-HURTADO FJ ${ }^{1}$, CAÑIZARES JM ${ }^{2}$
}

\begin{abstract}
RESUMEN
Objetivo: Registrar las características de la Retinopatía de la Prematuridad (RP) en una unidad neonatal a lo largo de 8 años, analizando los criterios del protocolo de despistaje y los factores de riesgo asociados.

Métodos: Estudio retrospectivo de los prematuros incluidos en el protocolo de despistaje. Evaluación de la sensibilidad de los criterios Peso al nacer (PN) $<1500$ gr, edad gestacional (EG) < 32 semanas y criterio subjetivo pediátrico (CSP) del protocolo y comparación estadística de la incidencia de RP y la necesidad de tratamiento entre los distintos grupos. Análisis estadístico entre los grupos con y sin RP de PN, EG, oxigenoterapia y patologías asociadas.

Resultados: Cuarenta de los 303 niños revisados presentaron RP $(13,2 \%)$. De ellos $31(77,5 \%)$ regresaron espontáneamente y $9(22,5 \%)$ requirieron tratamiento. Entre los 144 niños PN $<1.500$ gr la incidencia de RP fue $26,4 \%$ y entre los 159 niños con PN > 1.500 gr fue $1,3 \%$ ( $<<0,001)$. Se detectaron dos casos de RP con PN > 1500 gr y EG < 32 semanas, que no requirieron tratamiento. Hubo 84 casos $(27,7 \%)$ incluidos por CSP y en ninguno de ellos se
\end{abstract}

\begin{abstract}
Purpose: To assess the features of Retinopathy of Prematurity (ROP) in a Neonatal Intensive Care Unit during 8 years, analyzing the usefulness of the criteria in the screening protocol and the risk factors relating to the disease.

Methods: A retrospective study of the infants included in the screening program for ROP. The sensitivity of the criteria of birth weight $(\mathrm{BW})<1,500 \mathrm{~g}$, post-conceptional age (PCA) $<32$ weeks and subjective pediatric criteria (SPC) in the screening program were evaluated and compared with the incidence of ROP, and the need for treatment in these patient groups. Statistical analysis for ROP and noROP was then applied to the risk factors BW, PCA, oxygen therapy, and intercurrent diseases.

Results: Forty of 303 infants studied had ROP (13.2\%). In ROP cases, 31 (77.5\%) had spontaneous regression and $9(22.5 \%)$ needed treatment. The screening program included 144 children with BW $<1,500 \mathrm{~g}$ and 159 children with $\mathrm{BW}>1,500 \mathrm{~g}$. The incidence of ROP was $26.4 \%$ in the first group and $1.3 \%$ in the second group $(\mathrm{p}<0.001)$. Two cases were detected with a BW > 1,500 g but with a PCA
\end{abstract}

Recibido: 16/12/05. Aceptado: 18/5/06.

Hospital Virgen de las Nieves. Granada. España.

1 Doctor en Medicina. Servicio de Oftalmología.

2 Licenciado en Medicina. Servicio de Pediatría.

Correspondencia:

F.J. Rodríguez Hurtado

C/. Albero, 4

18014 Granada

España

E-mail: frhurtado@fundacionhvn.org 
detectó RP. Al aplicar la regresión logística a los factores de riesgo estudiados la única variable predictiva resultó ser el bajo peso.

Conclusiones: Ninguno de los niños incluidos en el protocolo con $\mathrm{PN}>1.500$ gr requirió tratamiento, pero usando sólo este criterio pueden no detectarse algunos casos de RP. La inclusión de niños por CSP debe restringirse.

Palabras clave: Retinopatía, prematuro, protocolo, factores de riesgo, peso al nacer, edad gestacional.

\section{INTRODUCCIÓN}

El número de recién nacidos prematuros ha aumentado en España en los últimos años hasta alcanzar la cifra del 7,1\% de todos los partos. Uno de los principales problemas de esos niños de bajo peso y baja edad gestacional es la Retinopatía del Prematuro (RP) y los neonatólogos están muy sensibilizados con su detección precoz, existiendo en todas las UCIs neonatales un protocolo de despistaje de la misma desde hace ya más de una década. Esta preocupación se extiende a los países donde está ocurriendo un incremento de la supervivencia de los prematuros (1). Como la detección precoz permite que estos niños se beneficien de una terapéutica eficaz, es fundamental un despistaje riguroso mediante fundoscopías binoculares bisemanales a todos los niños de Peso al Nacer (PN) inferior a 1.500 gr y Edad Gestacional (EG) inferior a 32 semanas. No obstante, ante el riesgo de que pase desapercibida una RP grave en prematuros de PN y EG superior a estos criterios la Academia Americana de Pediatría recomienda realizarlo también a todos los prematuros que los neonatólogos intensivistas consideren con factores de riesgo adicionales (2). Entre estos factores de riesgo se encuentra la intensa oxigenoterapia (3) y las enfermedades intercurrentes que puedan ocasionar fluctuaciones de la tensión de oxígeno en el tejido retiniano, ya que conllevarían una isquemia seguida de una fase proliferativa neovascular (4). La tendencia actual es la de limitar el protocolo a los dos principales criterios objetivos, incluso únicamente al PN $(5,6)$. En este trabajo analizamos estos criterios y también el Componente Subjetivo Pediátrico (CSP) del protocolo de despistaje de la RP en la Unidad de Cuida-
$<32$ weeks, and neither required treatment. There were 84 cases included because of SPC (27.7\%); no cases of ROP were detected in these. The only independent risk factor found in a multivariant analysis was birth weight.

Conclusions: None of the children included with a $\mathrm{BW}>1500 \mathrm{~g}$ required treatment for ROP, but several cases of ROP could be missed by using this criteria only. SPC must be restricted in the screening program (Arch Soc Esp Oftalmol 2006; 81: 275-280).

Key words: Retinopathy, prematurity, protocol, risk factors, birth weight, post-conceptional age.

dos Intensivos Neonatales de nuestro centro en un registro de 8 años. También hemos analizado la influencia de algunos factores de riesgo a los que los pediatras suelen acogerse para la inclusión subjetiva en el protocolo.

\section{SUJETOS, MATERIAL Y MÉTODOS}

Se ha realizado un estudio retrospectivo de los prematuros ingresados en nuestra Unidad Neonatal incluidos en el protocolo de despistaje de la ROP desde agosto de 1995 a diciembre de 2003. Dicho protocolo se elaboró en base a las directrices internacionales sugeridas por el estudio multicéntrico CRYO-ROP y recomendadas por prestigiosos hospitales de nuestro país $(7,8)$. Consistió en el examen oftalmoscópico bisemanal de todos los prematuros con $\mathrm{PN}<1.500$ gr o EG $<32$ semanas y de todos los prematuros incluidos en el grupo que hemos denominado Criterio Subjetivo Pediátrico (CSP): nacidos con PN y EG superiores a estas cifras pero que por su especial distrés respiratorio o sufrimiento postnatal fueron considerados de riesgo por los pediatras intensivistas. Hasta el año 2002 se inició el despistaje en la $6{ }^{a}$ semana postnatal, pero a partir de entonces se realiza desde la $4 .^{\text {a }}$ semana de vida o a las 31 semanas de edad postmenstrual y a los casos graves de RP se les realiza semanalmente. El examen fundoscópico fue realizado siempre por el mismo oftalmólogo (FRH) a lo largo de los 8 años. Como factores de riesgo adicionales se registraron los días de oxigenoterapia, la aparición de sepsis, necesidad de transfusiones sanguíneas, necesidad de cirugía intercurrente y aparición de hemorragia intracraneal. Para evaluar la sensibili- 
dad de los criterios de despistaje se realizó una comparación estadística de los grupos $\mathrm{PN}<1.500$ gr, $\mathrm{PN}>1500$ gr. y EG $<32$ semanas y $\mathrm{PN}>1.500$ gr y EG > 32 semanas (CSP) de la incidencia de RP y de la necesidad de tratamiento de la RP en ellos. Los estudios estadísticos se han realizado con el programa SPSS 11.0 para Windows, utilizando comparaciones bivariantes y análisis de regresión logística para los factores de riesgo entre los grupos con y $\sin$ ROP.

\section{RESULTADOS}

Cuarenta de los 303 niños revisados presentaron RP $(13,2 \%)$. De ellos 31 no alcanzaron el grado 3 plus y dejados a su evolución regresaron espontáneamente $(77,5 \%)$ y 9 mostraron signos de progresión en el grado 3 plus $(22,5 \%)$, requiriendo tratamiento. En el protocolo fueron incluidos 144 niños con PN $<1.500$ gramos y 159 niños con PN > 1500 gr. Usando sólo el criterio PN $<1.500$ gr se habrían detectado 38 casos de RP, lo que supone una sensibilidad del $95 \%$. La sensibilidad del $100 \%$ se obtuvo sumando los grupos PN $<1.500$ gr y EG $<32$ semanas. La distribución de casos con RP y casos normales según los distintos grupos de criterio de despistaje aparece en la tabla I. La incidencia de RP en niños con PN $<1.500 \mathrm{gr}$ fue del $26,4 \%$ y en niños con PN > 1.500 gr fue del 1,3\% (p<0,001). Los dos casos de RP del grupo PN > 1.500 gr presentaban una EG < 32 semanas, y alcanzaron como máximo un grado 2 en zona III, sin signos plus, regresando espontáneamente. Hubo 84 casos $(27,7 \%)$ incluidos por CSP y en ninguno de ellos se detectó RP. En el analisis bivariante de los factores de riesgo con variables cuantitativas existían diferencias significativas entre el grupo que presentó RP y el grupo sin RP para el PN y días de oxigenoterapia $(\mathrm{p}<0,05)$ pero no para la EG (tabla II). En el análisis bivariante de los factores de riesgo con variables cualitativas hubo asociación significativa del grupo con $\mathrm{RP}$ con sepsis y cirugía intercurrente respecto al grupo sin $\mathrm{RP}(\mathrm{p}<=0,05)$ (tabla III). Al aplicar la regresión logística la única variable independiente fue el bajo peso al nacer $(p<0,05)$.

Tabla III. Análisis de las variables cualitativas

\begin{tabular}{lrrrrr}
\hline Variables & \multicolumn{4}{c}{ ROP $(\mathrm{n}=40)$} & \multicolumn{3}{c}{$\begin{array}{r}\text { Sin ROP } \\
\text { N. }\end{array}$} & \multicolumn{1}{c}{ N. $^{\circ}$} & \multicolumn{1}{c}{$\%$} & \multicolumn{1}{c}{$\mathrm{p}$} \\
\hline HIC & 4 & $10,0 \%$ & 9 & $3,4 \%$ & 0,077 \\
Sepsis & 30 & $75.0 \%$ & 134 & $51,0 \%$ & 0,008 \\
Transfusión & 15 & $37,5 \%$ & 84 & $31,9 \%$ & ns \\
Cirugía & 7 & $17,5 \%$ & 15 & $5,7 \%$ & 0,016 \\
\hline \hline
\end{tabular}

HIC: Hemorragia intracraneal; Transfusión: Necesidad de una o más transfusiones; Cirugía: Intervención quirúrgica por patología intercurrente; Ns: no significativo.

Tabla I. Grupos de criterio de despistaje de la RP

\begin{tabular}{|c|c|c|c|c|}
\hline \multicolumn{5}{|c|}{ Criterios bajo peso y baja edad gestacional } \\
\hline & $<1.500 \mathrm{~g} \mathrm{y}<32 \mathrm{~s}$ & $<1.500 \mathrm{y}>32 \mathrm{~s}$ & $>1.500 \mathrm{~g} \mathrm{y}<32 \mathrm{~s}$ & $>1.500$ gr $y<32 \mathrm{~s}$ \\
\hline N. ${ }^{\circ} \operatorname{casos} \mathrm{RP}$ & 38 & 0 & 2 & 0 \\
\hline N. ${ }^{\circ}$ casos no RP & 136 & 8 & 75 & 84 \\
\hline \multicolumn{4}{|c|}{ Criterio bajo peso } & $\begin{array}{l}\text { Criterio subjetivo } \\
\text { pediátrico }\end{array}$ \\
\hline
\end{tabular}

RP: Retinopatía del prematuro; < 1.500 gr: Peso al nacer inferior a 1500 gr; $<32$ s: Edad gestacional inferior a 32 semanas.

Tabla II. Análisis de las variables cuantitativas

\begin{tabular}{|c|c|c|c|c|c|c|c|c|}
\hline & & & ROP & & & Sin ROP & & \\
\hline Variables & Medida & $\mathrm{N}$ & Media & D.T. & $\mathrm{N}$ & Media & D.T. & $\mathrm{p}$ \\
\hline Peso & Gramos & 261 & 998,8 & 294,3 & 40 & $1.688,1$ & 535,8 & $<0,001$ \\
\hline E.G. & Semanas & 261 & 27,8 & 2,1 & 40 & 32,02 & 2,9 & 0,06 \\
\hline $\mathrm{O}_{2}$ & Días & 225 & 5,6 & 9,7 & 36 & 34,3 & 41,3 & $<0,001$ \\
\hline
\end{tabular}

ROP: retinopatia del prematuro; N: número de casos; D.T.: desviacion típica; E.G: edad gestacional; $\mathrm{O}_{2}$ : oxigenoterapia. 


\section{DISCUSIÓN}

La incidencia de ROP resulta anormalmente baja en nuestra muestra $(13,2 \%)$ al haberse incluido un cuantioso grupo de niños (casi un tercio de ellos) que superaba las cifras objetivas recomendadas por la AAP. Cuando solo tenemos en cuenta a los niños con PN < 1.500 gr la incidencia de ROP sube al $26,6 \%$, cifra muy similar a la de los otros estudios realizados en hospitales españoles, que oscila entre el 26,2\% (9) y el 29,2\% (10). En el Hospital Karolinska de Suecia se ha publicado una incidencia del $40,1 \%$ de la población examinada (11). El CSP suele conllevar el examen de un exceso de niños que la medicina basada en la evidencia ha demostrado carentes de riesgo de RP (5). Por ello la tendencia actual es a usar como único criterio en el despistaje el $\mathrm{PN}<1.500$ gr. No obstante, no debe olvidarse que algunos niños con $\mathrm{PN}>1.500$ gr pueden presentar RP. Nosotros tuvimos dos casos, y aunque ambos regresaron espontáneamente, no conviene dejarlos pasar desapercibidos, sobre todo a raíz de la publicación de los resultados a largo plazo del estudio CRYO-ROP donde se detectaron algunos casos de desprendimiento de retina tardío en ojos no tratados (4). El dilema legal es si se puede renunciar al CSP recomendado por la Academia Americana de Pediatría para los niños con parámetros de PN y EG superiores a los límites no incluyendo en el despistaje a estos niños con «curso clínico inestable». El sentido común anima a examinarlos al menos una vez si han sido seleccionados por el neonatólogo, pero a la vez ese criterio no puede convertirse en el refugio de una medicina pediátrica defensiva basada en prejuicios sobre la oxigenoterapia (como ha ocurrido en nuestro CSP, donde la inmensa mayoría de esos casos han sido incluidos en cuanto se ha administrado algún grado de oxigenoterapia). Examinar fundoscópicamente en midriasis a prematuros que no lo necesitan es un gasto innecesario que no está exento de riesgos $(12,13)$. La tendencia actual es a disminuir más aún los límites de PN y EG en el protocolo, incluso a costa de dejar pasar desapercibidos casos leves de RP (14).

El bajo peso es determinante en la aparición de la ROP, como ha vuelto a ratificar este trabajo. La baja edad gestacional no presentó significación estadística, pero quedó cerca, y convendría reevaluarla con una mayor muestra. De los otros factores que influyen en la RP el más importante parece la oxigeno- terapia, existiendo numerosas recomendaciones para ajustarla a los mínimos necesarios, con objeto de conseguir una menor incidencia de formas graves $(15,16)$. Respecto a otros factores de riesgo, la septicemia se ha mostrado presente en la mayoría de nuestros casos graves, en coincidencia con lo sugerido por otros $(17,18)$. También la necesidad de cirugía durante las semanas cruciales para el desarrollo de la ROP ha mostrado ser un factor de gran influencia en nuestro estudio, aunque no hemos encontrado publicaciones al respecto. Otros factores de riesgo presentes en la literatura, tales como hemorragia intracraneal (19) o necesidad de transfusiones sanguíneas (20) no han tenido en nuestra muestra significación. Creemos que no existen evidencias suficientes para que los neonatólogos puedan añadir otro factor objetivo al bajo peso al nacer en los criterios de selección del grupo de despistaje.

\section{BIBLIOGRAFÍA}

1. Gibson DL, Sheps SB, Schetcher MT, Wiggins S, Mc Cormick $A Q$. Retinopathy of prematurity: a new epidemic? Pediatrics 1989; 83: 486-492.

2. American Academy of Pediatrics. Section on Ophthalmology: screening examination of premature infants for retinopathy of prematurity. Pediatrics 2001; 108: 809-811.

3. Askie LM, Henderson-Smart DJ. Early versus late discontinuation of oxygen in preterm or low birth weight infants. The Cochrane Library 2001; 4: 1-8.

4. Huchetson KA. Retinopathy of prematurity. Curr Opin Ophthalmol 2003; 14: 286-290.

5. Reynolds JD, Dobson V, Quinn GE, Fielder AR, Palmer EA, Hardy RJ, et al. Evidence-based screening criteria for retinopathy of prematurity: natural history data from the CRYO-ROP and LIGHT-ROP Studies. Arch Ophthalmol 2002; 120: 1470-1476.

6. Shalij-Delfos NE, Zijlmans BL, Wittebol-Post D, Tan KE, Cats BP. Screening for retinopathy of prematurity: do former guidelines still apply? J Pediatr Ophthalmol Strabismus 1996; 33: 35-38.

7. Fonseca Sandomingo A, Peralta Calvo J, Abelairas Gómez J. Retinopatía del prematuro. Madrid: Allergan; 1996.

8. Corcóstegui B. Retinopatía de la prematuridad. In: Corcóstegui B, Adán A, García-Arumi J, Mateo C, Nieto I. Cirugía vitreorretiniana. Indicaciones y técnicas. Madrid: Tecnimedia Editorial; 1999. I: 351-358.

9. Olea Vallejo JL, Corretger Ruhi FJ, Salvat Serra M, Frau Rotger E, Galiana Ferre C, Fiol Jaume M. Factores de riesgo en la retinopatia del prematuro. An Esp Pediatr 1997; 47: 172-175.

10. Martin Begue N, Perapoch López J. Retinopatía de la prematuridad: incidencia, severidad y resultados. An Pediatr (Barc) 2003; 58: 156-161. 
11. Holmstrom $G$, Broberger $U$, Thomassen P. Neonatal risk factors for retinopathy of prematurity - a population-based study. Acta Ophthalmol Scand 1998; 76: 204-207.

12. Rush R, Rush S, Nicolau J, Chapman K, Naqvi M. Systemic manifestations in response to mydriasis and physical examination during screening for retinopathy of prematurity. Retina 2004, 24: 242-245.

13. Aguirre Rodríguez FJ, Bonillo Perales A, Díez-Delgado Rubio J, González-Ripoll Garzón M, Arcos Martínez J, López Muñoz J. Paro cardiorrespiratorio relacionado con el exámen oftalmológico en prematuros. An Pediatr 2003; 58: 502-510.

14. Mathew MR, Fern AI, Hill R. Retinopathy of prematurity: are we screening too many babies? Eye 2002; 16: 538542.

15. Chow LC, Wright KW, Sola A; CSMC Oxygen Administration Study Group. Can changes in clinical practice decrease the incidence of severe retinopathy of prematu- rity in very low birth weight infants? Pediatrics 2003; 111: 339-345.

16. Rowlands E, Ionides AC, Chinn S, Mackinnon H, Davey $C C$. Reduced incidence of retinopathy of prematurity. $\mathrm{Br} J$ Ophthalmol 2001; 85: 933-935.

17. Brown BA, Thach AB, Song JC, Marx JL, Kwun RC, Frambach DA. Retinopathy of prematurity: evaluation of risk factors. Int Ophthalmol 1998; 22: 279-283.

18. Noyola DE, Bohra L, Paysse EA, Fernandez M, Coats DK. Association of candidemia and retinopathy of prematurity in very low birthweight infants. Ophthalmology 2002; 109: 80-84.

19. Christiansen SP, Fray KJ, Spencer T. Ocular outcomes in low birth weight premature infants with intraventricular hemorrhage. J Pediatr Ophthalmol Strabismus 2002; 39: 157-165.

20. Englert JA, Saunders RA, Purohit D, Hulsey TC, Ebeling M. The effect of anemia on retinopathy of prematurity in extreme low birth weight infants. J Perinatol 2001; 21: 21-26. 